\title{
ERRATUM
}

\section{A study on the association of cytochrome-P450 1A1 polymorphism and breast cancer risk in north Indian women}

\author{
Virendra Singh · Neeraj Rastogi · \\ Ashima Sinha - Abhai Kumar - Neeraj Mathur · \\ Mahendra Pratap Singh
}

Published online: 9 January 2007

(C) Springer Science+Business Media B.V. 2006

Erratum to: Breast Cancer Res Treat

DOI 10.1007/s10549-006-9264-2

Due to an unfortunate oversight the odds ratios and $p$-values for some genotypes were calculated the wrong way around. The right values are tabulated as follows.

\begin{tabular}{llll}
\hline & Genotype & $\begin{array}{l}\text { Odds ratio } \\
(95 \% \mathrm{CI})\end{array}$ & $p$-Value \\
\hline Total women & M1 (T/C) & $1.05(0.57-1.90)$ & 0.989 \\
& M1 (C/C) & $0.76(0.27-2.09)$ & 0.723 \\
& M2 (A/G) & $0.53(0.28-1.00)$ & 0.052 \\
& M4 (C/A) & $1.32(0.71-2.45)$ & 0.435 \\
Total premenopausal & M4 (A/A) & $4.96(0.47-246.91)$ & 0.179 \\
women & M1 (T/C) & $1.00(0.41-2.46)$ & 0.834 \\
& M2 (A/C) & $0.62(0.10-2.86)$ & 0.738 \\
Total postmenopausal & $0.98(0.40-2.41)$ & 0.865 \\
women & M1 (T/C) & $1.66(0.62-4.50)$ & 0.373 \\
& M1 (C/C) & $1.28(0.24-8.65)$ & 1.000 \\
& M2 (A/G) & $0.32(0.12-0.89)$ & 0.025 \\
& M4 (C/A) & $1.22(0.48-3.13)$ & 0.809 \\
\hline
\end{tabular}

The online version of the original article can be found at http:// dx.doi.org/10.1007/s10549-006-9264-2

V. Singh $\cdot$ A. Sinha $\cdot$ A. Kumar $\cdot$ N. Mathur .

M. P. Singh $(\bowtie)$

Industrial Toxicology Research Centre (ITRC), Mahatma

Gandhi Marg, Post Box 80, Lucknow 226001 UP, India

e-mail: singhmahendrapratap@rediffmail.com

N. Rastogi

Sanjay Gandhi Post Graduate Institute of Medical Sciences

(SGPGI), Lucknow 226 014, India 